\title{
Towards an in-depth characterization of Symbiodiniaceae in tropical giant clams via metabarcoding of pooled multi-gene amplicons
}

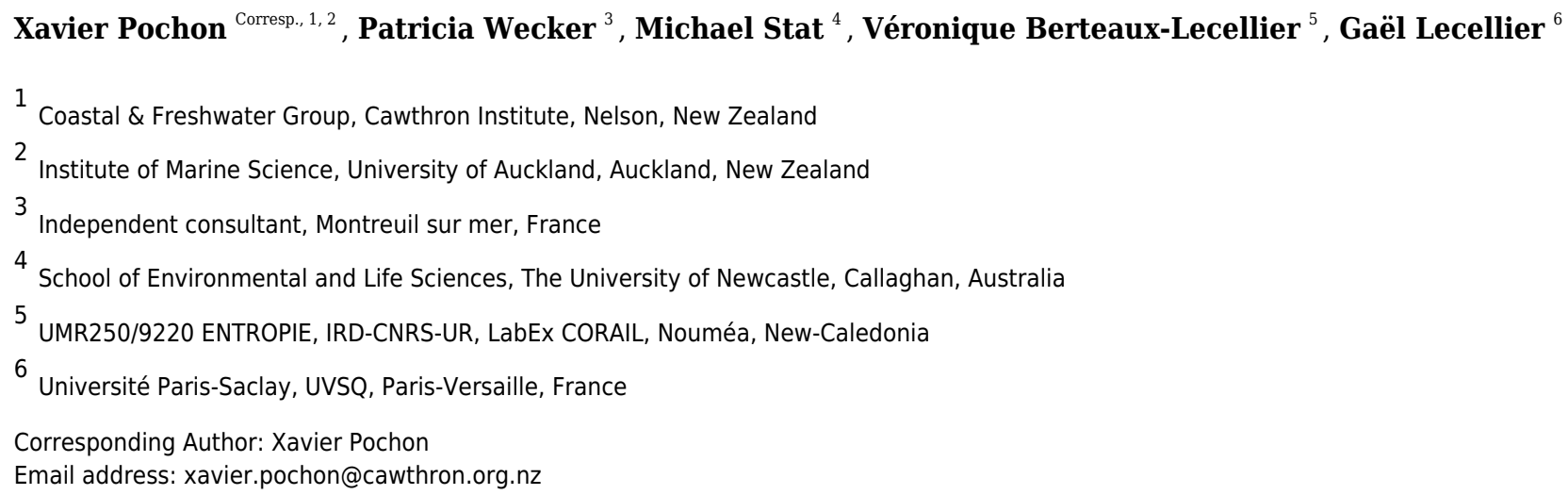

High-throughput sequencing is revolutionizing our ability to comprehensively characterize free-living and symbiotic Symbiodiniaceae, a diverse dinoflagellategroup that plays a critical role in coral reef ecosystems. Most studies however, focus on a single marker for metabarcoding Symbiodiniaceae, potentially missing important ecological traits that a combination of markers may capture. In this proof-of-concept study, we used a small set of symbiotic giant clam (Tridacna maxima) samples obtained from nine French Polynesian locations and tested a dual-index sequence library preparation method that pools and simultaneously sequences multiple Symbiodiniaceae gene amplicons per sample for indepth biodiversity assessments. The rationale for this approach was to allow the metabarcoding of multiple genes without extra costs associated with additional single amplicon dual indexing and library preparations. Our results showed that the technique effectively recovered very similar proportions of sequence reads and dominant Symbiodiniaceae clades among the three pooled gene amplicons investigated per sample, and captured varying levels of phylogenetic resolution enabling a more comprehensive assessment of the diversity present. The pooled Symbiodiniaceae multi-gene metabarcoding approach decribed here is readily scalable, offering considerable analytical cost savings while providing sufficient phylogenetic information and sequence coverage. 
1 Towards an in-depth characterization of Symbiodiniaceae in tropical giant clams via 2 metabarcoding of pooled multi-gene amplicons

3

4 Xavier Pochon ${ }^{1,2 *}$, Patricia Wecker ${ }^{3}$, Michael Stat ${ }^{4}$, Véronique Berteaux-Lecellier ${ }^{5}$, Gaël

$5 \quad$ Lecellier $^{5,6}$

6

$7 \quad{ }^{1}$ Coastal and Freshwater Group, Cawthron Institute, Nelson, New Zealand

$8 \quad 2$ Institute of Marine Science, University of Auckland, Auckland, New Zealand

$9 \quad{ }^{3}$ Independent consultant, Montreuil sur mer, France

$10{ }^{4}$ School of Environmental and Life Sciences, The University of Newcastle, Callaghan, Australia

$11{ }^{5}$ UMR250/9220 ENTROPIE, IRD-CNRS-UR, LabEx CORAIL, Nouméa, New-Caledonia.

$12{ }^{6}$ Université Paris-Saclay, UVSQ, Paris-Versailles, France

13

14

$15 *$ E-mail: xavier.pochon@cawthron.org.nz

16

17

18

19

20

21

22

23

24

25

Peer) reviewing PDF | (2019:01:34199:1:2:NEW 28 Mar 2019) 


\section{ABSTRACT}

28 High-throughput sequencing is revolutionizing our ability to comprehensively characterize free-

29 living and symbiotic Symbiodiniaceae, a diverse dinoflagellate group that plays a critical role in

30 coral reef ecosystems. Most studies however, focus on a single marker for metabarcoding

31 Symbiodiniaceae, potentially missing important ecological traits that a combination of markers

32 may capture. In this proof-of-concept study, we used a small set of symbiotic giant clam (Tridacna

33 maxima) samples obtained from nine French Polynesian locations and tested a dual-index

34 sequence library preparation method that pools and simultaneously sequences multiple

35 Symbiodiniaceae gene amplicons per sample for in-depth biodiversity assessments. The rationale

36 for this approach was to allow the metabarcoding of multiple genes without extra costs associated

37 with additional single amplicon dual indexing and library preparations. Our results showed that

38 the technique effectively recovered very similar proportions of sequence reads and dominant

39 Symbiodiniaceae clades among the three pooled gene amplicons investigated per sample, and

40 captured varying levels of phylogenetic resolution enabling a more comprehensive assessment of

41 the diversity present. The pooled Symbiodiniaceae multi-gene metabarcoding approach decribed

42 here is readily scalable, offering considerable analytical cost savings while providing sufficient

43 phylogenetic information and sequence coverage. 


\section{INTRODUCTION}

48 Giant clams (Family Tridacnidae) play important roles in reef systems, acting as shelter for a

49 number of organisms (Cabaitan et al. 2008; Mercier \& Hamel 1996), contributing to primary

50 production through their symbiosis with dinoflagellates (Neo et al. 2015), and as effective filter

51 feeders (Klumpp \& Griffiths 1994). Due to their large size, relative abundance and longevity, giant

52 clams can be considered as centennial barometers of reef health (Knop 1996; Romanek \&

53 Grossman 1989; Watanabe et al. 2004). Unfortunately, as a highly prized resource throughout

54 much of their Indo-Pacific range, the family Tridacnidae also contains some of the most

55 endangered species due to overfishing, i.e. wild stock depletion and local extinctions (IUCN Red

56 List 2018). This collapse is compounded with habitat degradation (Bin Othman et al. 2010).

58 Giant clams on shallow reefs allow for the establishment of a diverse in-situ reservoir of interacting

59 fungal, bacterial, and micro-algal communities (Baker 2003; Neo et al. 2015). Importantly, they

60 form obligatory symbioses with, and release living cells of, Symbiodiniaceae sensu LaJeunesse et

61 al. (2018), a group of dinoflagellates that are critical for the survival of a myriad of tropical

62 invertebrates, including corals. Despite these dynamic interactions, very little is known about the

63 extent of symbiont diversity within giant clams and the potential exchange with other reef

64 invertebrates engaged in similar symbiotic associations (i.e. nudibranchs and corals; Wecker et al.

65 2015). Unlike traditional molecular techniques (e.g. PCR-based fingerprinting methods and

66 Sanger sequencing) that have been extensively used to shed light on Symbiodiniaceae diversity in

67 reef organisms (reviewed in Coffroth \& Santos 2005; Stat et al. 2006), recent advances in High-

68 Throughput Sequencing (HTS) technologies now enable unprecedented sequencing depth for

69 global biodiversity assessments of symbiotic and free-living communities of Symbiodiniaceae 
70 (Boulotte et al. 2016; Cunning et al. 2015; Edmunds et al. 2014; Hume et al. 2018; Shinzato et al.

71 2018; Thomas et al. 2014). Nevertheless, such studies usually focus on metabarcoding analyses of

72 single molecular markers in isolation, in particular the ITS2 marker (but see Smith et al. 2017;

73 Thomas et al. 2014), potentially overlooking intrinsic phylogenetic differences known to occur

74 between distinct Symbiodiniaceae genes (Pochon et al. 2012; Pochon et al. 2014).

A variety of HTS library preparation methods exist for metabarcoding biological samples using Illumina ${ }^{\mathrm{TM}}$ sequencing platforms, including the use of fusion tag primers (Elbrecht \& Steinke 2018; Stat et al. 2017), the ligation of Illumina ${ }^{\mathrm{TM}}$ adapters using TruSeq ${ }^{\mathrm{TM}}$ PCR-free kits (Rhodes et al. 2014), and the addition of Illumina ${ }^{\mathrm{TM}}$ adapters via dual-index sequencing (Kozich et al. 2013). The latter technique requires two distinct rounds of PCR analyses. The first round uses gene-specific primers modified to include Illumina ${ }^{\mathrm{TM}}$ adapter tails. Following purification of the PCR products, a second short round of PCR is applied using Nextera ${ }^{\mathrm{TM}}$ library construction kits that involve individual primer sets containing the Illumina ${ }^{\mathrm{TM}}$ adapter and sequencing primer sequence. This second PCR step is usually performed on individual PCR amplicon products before the pooling and sequencing of multiple samples so that demultiplexing of sequence data results in appropriate identification of input samples. For laboratories that use the services of external genomic facilities for the preparation of their dual-index libraries, an increased sample set usually correlates positively with the analytical cost due, in part, to the use of additional Nextera ${ }^{\mathrm{TM}}$ indexed primers. Therefore, one solution for reducing costs when performing multi-gene analyses of individual samples, is to pool the PCR amplicon products prior to the second PCR step, followed by the sequencing and gene-specific demultiplexing per sample. 
93 Here we conducted a preliminary assessment of a dual-index multi-gene metabarcoding approach

94 via the pooling and side-by-side HTS analysis of PCR amplicons from three commonly employed

95 nuclear and chloroplastic Symbiodiniaceae markers. The ability to combine multiple gene

96 amplicon targets per sample offers considerable analytical cost savings while providing sufficient

97 phylogenetic information and sequence coverage. This study describes a multi-marker

98 metabarcoding approach using giant clam Tridacna maxima as a model and discusses future 99 applications for improving analyses of coral reef holobionts.

\section{MATERIAL AND METHODS}

\section{Sample collection and DNA extraction}

103 For this study, twelve DNA extracts from Tridacna maxima biopsies, previously collected 104 between February $1^{\text {st }} 2011$ and November $2^{\text {nd }} 2013$ from nine islands in the French Polynesian 105 Archipelagos (Figure 1, Table S1) were used (Dubousquet et al. 2018).

\section{Preparation of Pooled Amplicons High-Throughput Sequencing Libraries}

108 Three sets of Symbiodiniaceae-specific primers with Illumina ${ }^{\mathrm{TM}}$ adapter tails (Table S2) were

109 used to amplify each sample (S141-S152; Table 1) in separate Polymerase Chain Reactions (PCR).

110 Three markers were amplified: (i) the Internal Transcribed Spacer 2 (ITS2) of the nuclear

111 ribosomal RNA array using primers ITSD_illu and ITS2rev2_illu, (ii) the D1-D2 region of the

112 28S large subunit ( $L S U$ ) nuclear ribosomal RNA gene using the newly designed primers

113 LSU1F_illu and LSU1R_illu, and (iii) the hyper-variable region of the chloroplast 23S (23S)

114 ribosomal RNA gene using primers 23SHyperUP_illu and 23SHyperDN_illu (Manning \& Gates

115 2008; Pochon et al. 2010). The new forward and reverse LSU primers were designed within the 
116 conserved areas flanking the D1-D2 region of a previously published LSU sequence alignment

117 (Pochon et al. 2012; Figure S1; Supplementary File 1), containing 93 sequences of

118 Symbiodiniaceae (with representatives from all nine existing clades), as well as 8 sequences from

1193 dinoflagellate species represented by Gymnodinium simplex, Pelagodinium beii, and Polarella

120 glacialis. Primers were designed to be 'dinoflagellate-specific' using MacVector v11.0.2

121 (MacVector Inc., NC, USA), avoiding cladal bias and minimizing self/duplex hybridization and

122 internal secondary structure problems (Figure S1).

123

124 PCR was performed for each sample and for each gene separately in $50 \mu \mathrm{L}$ volumes, with the 125 reaction mixture containing $45 \mu \mathrm{L}$ of Platinum PCR SuperMix High Fidelity (Life Technologies),

$12610 \mathrm{uM}$ of each primer, and $10-20 \mathrm{ng}$ of template DNA. In order to maximize specificity to

127 Symbiodiniaceae, a touchdown PCR protocol was used for each reaction as follows: (i) $95{ }^{\circ} \mathrm{C}$ for

$12810 \mathrm{~min}$; (ii) 25 cycles of $94{ }^{\circ} \mathrm{C}$ for $30 \mathrm{~s}, 65^{\circ} \mathrm{C}$ for $30 \mathrm{~s}$ (decreasing the annealing temperature 0.5

$129{ }^{\circ} \mathrm{C}$ for every cycle after cycle 1), and $72{ }^{\circ} \mathrm{C}$ for $1 \mathrm{~min}$; (iii) 14 cycles of $94{ }^{\circ} \mathrm{C}$ for $30 \mathrm{~s}, 52^{\circ} \mathrm{C}$ for

$13030 \mathrm{~s}$ and $72{ }^{\circ} \mathrm{C}$ for $1 \mathrm{~min}$; and (iv) a final extension of $72{ }^{\circ} \mathrm{C}$ for $10 \mathrm{~min}$. Amplicons of the correct

131 size (estimated visually via gel electrophoresis) were purified using Agencourt AMPure XP PCR

132 Purification beads following the manufacturers' instructions. In order to sequence the three gene

133 amplicons per sample collectively using HTS, individual purified products for each marker

134 originating from the same giant clam were pooled together to enable the attachment of the same

135 Nextera $^{\mathrm{TM}}$ index (i.e. 12 samples). This was achieved by quantifying the amplicons using a Qubit

136 Fluorometer 2.0 (Life Technologies), diluting to $1 \mathrm{ng} / \mu \mathrm{L}$ using Milli-Q water and mixing $5 \mu \mathrm{L}$ of

137 each of gene amplicon from the same giant clam together. To assess the levels of cross-

138 contamination between samples potentially arising during the library indexing step, nine unmixed 
139 amplicon products (i.e. ITS2, $L S U$ and $23 S$ amplicons from three haphazardly selected giant clams;

140 samples S141-S143; Table 1), each with their own unique index to be added, were also prepared.

142 The resulting 21 samples were placed on a 96-well plate along with other samples published 143 elsewhere (Zaiko et al. 2016), and sent to New Zealand Genomics Ltd. (University of Auckland,

144 New Zealand) for HTS library preparation which involved a second round of PCR to attach the 145 Nextera $^{\mathrm{TM}}$ indexes on to the amplicons for MiSeq Illumina ${ }^{\mathrm{TM}}$ sequencing. PCR products were 146 combined in equimolar concentrations and the final library paired-end sequenced on an Illumina ${ }^{\mathrm{TM}}$

147 MiSeq using a 500 cycle $(2 \times 250)$ MiSeq ${ }^{\circledR}$ v2 Reagent Kit and standard flow cell.

\section{Bioinformatics}

150 Illumina ${ }^{\mathrm{TM}}$ sequence datasets were prepared using the read preparation and dereplication 151 pipeline of USEARCH (Edgar 2010). Firstly, paired reads were merged (fastq mergepairs 152 command) and filtered (fastq filter command) with an expected number of error of 0.25 . More

153 than $90 \%$ of the base pairs had a Q score $>40$. Next, samples were demultiplexed in three groups, 154 primers were trimmed and a global trimming was operated according to the recommendations for 155 ITS amplicon reads (Edgar 2013). The sequence data were dereplicated and unique singletons 156 found across the complete dataset were discarded.

158 For phylogenetic assignments of Symbiodiniaceae, three distinct annotated reference databases 159 (ITS2, LSU and 23S) were generated in fasta format, including sequence representatives from each 160 of nine Symbiodiniaceae clades (A to I), with (i) 409 representative ITS2 phylotypes from 161 GeoSymbio (Franklin et al. 2012), (ii) 37 representative $L S U$ sequences from Pochon et al. (2012), 
162 and (iii) 104 sequences of $23 S$ from Takabayashi et al. (2011). The three reference sequence

163 databases used in the present study are provided in the Supplementary File 1. Symbiodiniaceae

164 assignments were performed using the software 'Kallisto' (Bray et al. 2016) which provides speed

165 and accuracy for optimal analysis of large-scale datasets (e.g. large RNA-Seq data) without the

166 need for time-consuming alignment steps.

167

168 Because the main goal of the present pilot study was to investigate the sequencing depth and

169 potential inter-marker biases of the multi-marker metabarcoding approach using giant clam

170 samples as a proof-of-concept, as opposed to describing potentially novel Symbiodiniaceae

171 diversity in these samples, we modified the Kallisto pipeline to only retain HTS reads yielding

172 exact matches (i.e. without ambiguity amongst k-mers) to individual referenced genotypes in each

173 gene. Individual sequences generated via HTS were then blasted against all pseudo-alignments

174 and exact matches against the entire population of k-mers are recorded. To reduce mis-

175 assignments, all merged reads with ambiguities between k-mers of different reference genotypes

176 were determined as chimeric and removed from the dataset. These sequences that did not result in

177 exact matches could correspond to non-Symbiodiniaceae sequences or to sequences not comprised

178 in our custom database. Therefore, a second comparison using BLASTn (threshold: e-value $<10^{-}$

$179{ }^{30}$ ) against the National Center for Biotechnology Information (NCBI) nucleotide databases was

180 performed and the accession numbers yielding exact matches were retained for downstream

181 analyses. The number of unique sequences matching genotypes in the reference databases and

182 GenBank was recorded (Table S3). Raw sequence data were submitted to the BioProject Archive 183 under accession PRJNA471926 (SRR7181922-SRR7181942). 


\section{Sequence Diversity Analyses}

186 Unique sequence genotypes found at or above a $0.05 \%$ threshold from the total sequence

187 abundance per sample were scored (Table S3) and the specific genotypes of reference (i.e. from

188 in-house reference databases and GenBank) were retained for sequence diversity and phylogenetic

189 analyses. Global sequence diversity from each of the three datasets $(23 S, I T S 2$, and $L S U)$ were

190 visualized using the plug-in DataBurst implemented in Excel (Microsoft Office version 2013 or

191 later).

192

193 One sequence alignment was generated for each of the three investigated gene datasets using the 194 sequence alignment software BioEdit v7.2.5 (Hall 1999). Owing to the difficulty in aligning 195 sequences from Symbiodinium (clade A) and Cladocopium (clade C) genera when using the 23S 196 and ITS2 genes, and between Symbiodiniaceae and non-Symbiodiniaceae (i.e. clams, fungi, and

197 plants) sequences, phylogenetic reconstructions only aimed at depicting pair-wise relationships 198 between retained sequence genotypes. Therefore, unrooted phylogenetic inferences were 199 generated using the neighbor-joining method implemented in the program MEGA v. 7.0 (Kumar 200 et al. 2016), with the p-distance model and gaps treated as pairwise deletions. Internal nodes 201 support was tested using the bootstrap method (Felsenstein 1985) and 500 replicates.

\section{RESULTS}

A total of 1,590,047 sequences were obtained from the 21 samples $(75,716+/-41,576$ 205 sequences per sample), which included 12 amplicon samples (S141-S152) each containing three pooled gene products $(23 S, I T S 2$, and $L S U)$ and nine amplicon samples from three selected giant clam isolates (S141, S142, and S143) which only contained a single gene amplicon as internal 
208 controls (Table 1; Table S3). One sample (internal control S143 for ITS2) failed the sequencing 209 step with only 130 raw reads produced. After read cleaning, the total number of high-quality 210 sequences was $1,104,687(52,604+/-29,250$ sequences per sample). The proportion of total reads

211 (Table 1) between the three investigated genes was well-balanced with 398,442 reads (23S), 212339,780 reads (ITS2), and 359,768 reads (LSU). In contrast, unique reads varied between 23,779

213 sequences for the $23 S$ gene and 71,776 sequences for the $L S U$ gene (Table S3). The inclusion of 214 nine positive controls, representing three amplicon products per gene sequenced in isolation, 215 revealed the presence of low levels of sequence cross-contamination between samples (mean of 4.5 sequences $\pm 4.6 \mathrm{SD}$ ) (Table 1). This low-level of background contamination (1 to 23 sequences

217 per sample) represented $<0.003 \%$ of the total reads per sample (Table S3). Therefore, as a 218 conservative measure, we chose to remove sequences that represented $<0.05 \%$ of the total 219 sequence abundance per sample.

220

221 Our bioinformatics pipeline identified 43 Symbiodiniaceae $23 S$ chloroplast genotypes, including 22216 that matched the $23 S$ reference database and another 27 that matched sequences in GenBank. 223 After exclusion of genotypes represented by less than $0.05 \%$ of the sequence abundance in each 224 sample (Table S3), the number of unique $23 S$ Symbiodiniaceae sequences retained for 225 phylogenetic analysis was eleven, including six sequences matching the $23 S$ in-house reference 226 (Figure S2; Table S3). Similarly, blasting ITS2 and LSU datasets against both types of databases 227 led to the identification of 117 and 93 unique sequences when using the original datasets, and to 22846 and 51 unique sequences following the $0.05 \%$ filtering threshold, respectively. 
230 Diversity diagrams were generated to visualize the sequence abundance of Symbiodiniaceae

231 generic and sub-generic sequences recovered from the twelve giant clam samples and among the

232 three investigated genes (Figure 2). The pooled multi-gene approach yielded similar proportions

233 of dominant genera, but with some notable differences. The genus Symbiodinium (previously

234 Clade A) dominated in all three markers, particularly in $23 S(91.8 \%$; dominant sub-generic

235 sequence chvA2), with lower but similar proportions between ITS2 (81.7\%; dominant sub-generic

236 sequences A3/A6) and $\operatorname{LSU}(83.9 \%$; dominant sub-generic sequences A3/A13). The genus

237 Cladocopium (previously Clade C) represented 7.9\% (dominant sub-generic sequence chvC1),

$23818.2 \%$ (dominant sequence $\mathrm{C} 1$ ), and $15.0 \%$ (dominant sequence $\mathrm{C} 1$ ) of reads for the $23 S$, ITS2,

239 and $L S U$ markers, respectively. Geracladium (previously clade G) was only detected using the

240 chloroplast $23 S$ gene ( $0.2 \%$ of reads), whereas the nuclear $L S U$ gene displayed reduced specificity

241 for Symbiodiniaceae as indicated by $\sim 1 \%$ of sequence reads matching other organisms such as

242 streptophytes (Mitchella repens and Asclepias verticillata), and the host giant clam T. maxima.

243 Overall, the proportion of dominant Symbiodiniaceae generic and sub-generic sequences

244 recovered between the pooled samples and the positive (single gene) controls were very similar

245 (Table 2).

246

247 DISCUSSION

248 Multi-gene metabarcoding: more for less

249 The concept of pooled multi-gene amplicons for dual-indexed metabarcoding, i.e. the tagging and

250 pooling of distinct gene amplicons before Illumina ${ }^{\mathrm{TM}}$ adapter indexing and simultaneous

251 sequencing of samples, has been used in other research fields (e.g. Elbrecht \& Steinke 2018;

252 Keeley et al. 2018; Marcelino \& Verbruggen 2016; von Ammon et al. 2018; Zhang et al. 2018), 
253 but has never been applied to Symbiodiniaceae dinoflagellates. In this proof-of-concept study, we

254 show that the technique effectively recovered similar proportions of sequence reads and

255 Symbiodiniaceae genera among the three pooled genes investigated per sample, providing more

256 confidence that single gene primer biases did not occur during Nextera ${ }^{\mathrm{TM}}$ indexing. Another

257 advantage is the ability to simultaneously visualize varying levels of phylogenetic resolution,

258 enabling a more comprehensive assessment of the diversity present. For example, while the

259 traditional 'species-level' ITS2 marker (LaJeunesse 2001) enabled characterization of 46

260 Symbiodiniaceae sub-generic sequences, the $L S U$ marker, interestingly, offered both a similarly

261 high resolution for Symbiodiniaceae (46 sub-generic sequences) and a reduced specificity by also

262 enabling identification of other host-associated organisms such as streptophytes, as well as the host

263 Tridacna. Nevertheless, the results regarding the streptophytes need to be interpreted with caution.

264 Indeed, the herbaceous woody shrub Mitchella repens and the milkweed Asclepias verticillata are

265 both land plants restricted to the eastern coasts of North and South America and are, therefore,

266 highly unlikely to represent true detections from our giant clam samples. It is possible that they

267 are either the result of PCR contamination, inaccurate annotation in GenBank and/or correspond

268 to the next sequence hit, the plant Coffea arabica which has a wide distribution including the

269 Pacific region. The hyper-variable region of the chloroplast $23 S$ marker used here is more

270 conserved than the ITS2 and $L S U$ regions, but has been successfully used for specifically targeting

271 low abundance free-living Symbiodiniaceae cells from environmental samples (Decelle et al.

272 2018; Manning \& Gates 2008; Pochon et al. 2010; Takabayashi et al. 2011). The unique detection

273 of Gerakladium (clade G) using the 23S marker highlights the added value of the multi-gene

274 approach for broader Symbiodiniaceae screening efficiency. This marker also showed remarkable

275 consistency in the proportion of recovered sub-generic types between 'Pooled' and 'Control' 
276 samples, and contrasted with the ITS2 and LSU markers (Table 2). For example, four ITS2

277 sequences were detected in the 'Pooled' but not in the 'Control' samples, and there were five

278 instances where $L S U$ sequences were detected in the 'Control' but not in the 'Pooled' samples.

279 Similarly, another difficult-to-explain contrast was observed for samples S147 and S152 (Figure

280 S3) where the proportion of recovered Symbiodiniaceae genera differed markedly between $23 S$

281 and ITS2/LSU Markers. Although the above minor differences are likely attributable to PCR or

282 sequencing biases, further research applying similar multi-gene approaches would improve our

283 understanding of the intrinsic characteristics of these commonly employed Symbiodiniaceae

284 markers and help guide the interpretation of such datasets.

285

286 Analytical cost is an important consideration for any research group aiming to monitor coral reef

287 ecosystems, and the budget needed to include HTS for biodiversity assessments is highly variable.

288 The cost depends on the number of gene regions investigated, method of library preparation,

289 sequencing depth, and whether pooling amplicons is employed as shown here. A comparative cost

290 estimate between the pooling of three PCR amplicons for the twelve investigated samples versus

291 the complete processing of thirty six individual PCR amplicons showed that the pooling method

292 enabled an approximately 5.4 times cost saving on reagents (tubes, tips, purification/quantification,

293 and Nextera ${ }^{\mathrm{TM}}$ indexing). In this context, our approach is readily scalable and has the potential to

294 offer substantial savings in terms of both time and cost, for example, by enabling coral reefs

295 researchers to generate multi-gene Symbiodiniaceae data in a 96-well format for the price of a

296 single dual-indexed Illumina ${ }^{\mathrm{TM}}$ MiSeq run. Nevertheless, the caveat is that upscaling this pooling

297 method beyond a certain threshold will inevitably lead to a decrease in sequencing depth per

298 sample per gene. Exceeding this threshold may be problematic for researchers wanting to gather a 
299 complete overview of fine-scale diversity, or study potential low-frequency intragenomic variants.

300 Further research is needed to better understand this tradeoff and to set appropriate thresholds.

301 Another important consideration is to make sure that the distinct pooled amplicons are of similar

302 base-pair length, otherwise shorter gene amplicons may generate more sequence reads than the

303 longer co-occurring amplicons (Engelbrektson et al. 2010). Additional studies are also required to

304 investigate whether multiplexing i.e. the mixing of multiple primer sets in the original PCR to

305 produce multi-gene amplicons (De Barba et al. 2014; Fiore-Donno et al. 2018) would result in

306 similar proportions of Symbiodiniaceae genotypes between markers such as shown in the present

307 study. Such an approach, if validated, would allow very significant additional cost savings.

\section{Paving the way for comprehensive biodiversity assessment of giant clams}

310 Giant clams on shallow reefs allow for the establishment of a diverse in-situ reservoir of

311 interacting fungal, bacterial, and micro-algal communities (Baker 2003; Neo et al. 2015). For

312 example, they commonly harbor Symbiodiniaceae from at least three distinct genera

313 (Symbiodinium [clade A], Cladocopium [clade C], and/or Durusdinium [clade D]) simultaneously

314 or in isolation within one host, with Symbiodinium being the dominant symbiont genus in most

315 clams (Baillie et al. 2000; DeBoer et al. 2012; Ikeda et al. 2017; Ikeda et al. 2016; Pappas et al.

316 2017; Trench et al. 1981). Similar to coral symbiosis, it is assumed that the genotypic composition

317 of Symbiodiniaceae in giant clams is influenced by environmental or physical parameters (e.g.

318 temperature, irradiance), or by life stages and taxonomic affiliation (Ikeda et al. 2017; Pappas et

319 al. 2017). Giant clam larvae (veliger) acquire free-living Symbiodiniaceae cells 'horizontally' from

320 their surrounding environment (Fitt \& Trench 1981). When mature, giant clams (e.g. Tridacna

321 derasa) expel high numbers of intact symbionts in their faeces at rates of $4.9 \times 10^{5}$ cells d$^{-1}$ (Buck 
322 et al. 2002; Maruyama \& Heslinga 1997). Despite the dynamic interaction of symbionts between

323 Tridacnidae and the environment, very little is known about the extent of symbiont diversity within

324 giant clams and the potential exchange with other reef invertebrates engaged in similar symbiotic

325 associations.

326

327 In this preliminary study, we found that genera Symbiodinium (clade A) and Cladocopium (clade

328 C) dominated in adult giant clams in French Polynesia (Figure S3). Symbiodinium was the major

329 genus in our samples and in particular the closely related sub-generic ITS2 genotypes A3 and A6,

330 previously described as Symbiodinium tridacniadorum, and therefore associated

331 with Tridacna clams (Lee et al. 2015). A3 is the most dominant genotype in T. maxima around the

332 world and both A3/A6 are more likely to be sampled in giant clams from shallow reefs (Weber 333 2009).

334

335 Furthermore, for Cladocopium we found that the generalist ITS2 genotype C1 (LaJeunesse et al. 336 2003) co-dominated in our samples, which is consistent with a previous study showing $\mathrm{C} 1$ as a 337 common genotype in T. maxima from around the world (Weber 2009). We also found a smaller 338 percentage of C3z, Cspd and C50 ITS2 genotypes, which to our knowledge have not yet been 339 found in T. maxima, and are usually restricted to corals (LaJeunesse et al. 2004; LaJeunesse et al. 340 2010; Macdonald et al. 2008; Shinzato et al. 2018). Finally, we did not detect any symbiont from

341 the genus Durusdinium (Clade D) despite in-depth sequencing. However, Durusdinium has never

342 been detected in T. maxima from French Polynesia compared to other regions such as the Indian

343 Ocean (DeBoer et al. 2012; Weber 2009). As we only worked with adult clams from shallow water,

344 it would be interesting to confirm the hypotheses of Ikeda et al. (2017) and Weber (2009) who 
345 argued that Durusdinium symbionts might be restricted to 'young' T. squamosa clams (less than

$34611 \mathrm{~cm}$ ) or that giant clams harbored this dinoflagellate genus only when sampled from deeper

347 reefs, respectively. Nevertheless, the small dataset used in the present study precludes us from

348 making any relevant assumptions about potentially novel symbiont diversity in giant clams. In

349 particular, the use of the Kallisto bioinformatics pipeline which restricted the analysis to $100 \%$

350 sequence similarity hits is likely not suitable for the many studies where a high degree of sequence

351 novelty is found. Additionally, a weakness of the Kallisto method is that the analysis of k-mers

352 that are poorly divergent and/or not well represented in the reference database may impact the final

353 sequence annotation, in particular at the sub-clade level. For example, the ITS2 genotype C1 was

354 only detected following NCBI blast, even though this sequence was present in our in-house

355 database. This is not ideal, and one could argue that sequences should have been blasted

356 exclusively against GenBank. Nevertheless, the chosen bioinformatics pipeline did not affect the

357 general findings of the present study and was appropriate for this purpose. It is our hope, however,

358 that our multi-gene approach will be investigated further using a more comprehensive giant clam

359 dataset along with the development of an alternative bioinformatics method guiding users on the

360 assignment of genus to species-level taxon ID to novel multi-gene sequences for deposition to

361 GenBank.

362

363 CONCLUSIONS

364 This pilot project explored the use of pooled amplicon metabarcoding for rapid, cost-effective and

365 in-depth characterization of Symbiodiniaceae dinoflagellates using the giant clam T. maxima as a

366 model. Our results showed that the technique effectively recovered similar proportions of sequence

367 reads and Symbiodiniaceae diversity among the three gene amplicons investigated per sample 
368 enabling a more comprehensive assessment of the diversity present, while also offering

369 appreciable analytical cost savings. We also found that Symbiodinium (clades A) and Cladocopium

370 (clade C) were the dominant genera in adult giant clams in French Polynesia, with similar sub-

371 generic genotypes (ITS2 A3, A6, and C1) previously described as commonly associated with giant

372 clams from around the world. Our approach paves the way for more comprehensive surveys of

373 this important yet endangered group of reef invertebrates and its potential role as an important

374 Symbiodiniaceae reservoir for declining coral reefs. More work is required to test the applicability

375 of this method to other symbiotic organisms as well as to environmental samples. Future

376 investigations may also expand on this method to clarify species-level differentiation among

377 Symbiodiniaceae taxa using other markers (e.g. nuclear Actin, chloroplast $p s b A$ ), or

378 simultaneously characterize all organisms (viruses, bacteria, fungi, and other eukaryotes)

379 associated with a more diverse host range. Such holistic diversity assessments will improve our

380 knowledge on the ecology and evolution of tropical holobionts and better predict the adaptation of

381 coral reefs in a rapidly changing environment.

382

\section{ACKNOWLEDGMENTS}

384 We thank Jonathan Drew for his support in the laboratory, and Charley Waters, Sam Murray and

385 Chris Cornelisen for valuable discussions during manuscript preparation.

386

\section{REFERENCES}

388

389

390

391

392

393
Baillie BC, Belda-Baillie CA, Silvestre V, Sison M, Gomez AV, Gomez ED, and Monje V. 2000. Genetic variation in Symbiodinium isolates from giant clams based on random-amplifiedpolymorphic DNA (RAPD) patterns. Marine Biology 136:829-836.

Baker AC. 2003. Flexibility and specificity in coral-algal symbiosis: diversity, ecology, and biogeography of Symbiodinium. Annual Review of Ecology, Evolution, and Systematics 34:661-689. DOI 10.1146/annurev.ecolsys.34.011802.132417 
394

395

396

397

398

399

400

401

402

403

404

405

406

407

408

409

410

411

412

413

414

415

416

417

418

419

420

421

422

423

424

425

426

427

428

429

430

431

432

433

434

435

436

437

438

439
Bin Othman AS, Goh GHS, and Todd PA. 2010. The distribution and status of giant clams (Family Tridacnidae) - A short review. Raffles Bulletin of Zoology 58:103-111.

Boulotte NM, Dalton SJ, Carroll AG, Harrison PL, Putnam HM, Peplow LM, and van Oppen MJ. 2016. Exploring the Symbiodinium rare biosphere provides evidence for symbiont switching in reef-building corals. ISME Journal

DOI 10:2693-2701. 10.1038/ismej.2016.54

Bray NL, Pimentel H, Melsted P, and Pachter L. 2016. Near-optimal probabilistic RNA-seq quantification. Nature Biotechnology 34:525-527. DOI 10.1038/nbt.3519

Buck BH, Rosenthal H, and Saint-Paul U. 2002. Effect of increased irradiance and thermal stress on the symbiosis of Symbiodinium microadriaticum and Tridacna gigas. Aquatic Living Resources 15:107-117.

Cabaitan PC, Gomez ED, and Aliño PM. 2008. Effects of coral transplantation and giant clam restocking on the structure of fish communities on degraded patch reefs. Journal of Experimental Marine Biology and Ecology 357:85-98.

DOI 10.1016/j.jembe.2008.01.001

Coffroth MA, and Santos SR. 2005. Genetic diversity of symbiotic dinoflagellates in the genus Symbiodinium. Protist 156:19-34. DOI 10.1016/j.protis.2005.02.004

Cunning R, Yost DM, Guarinello ML, Putnam HM, and Gates RD. 2015. Variability of Symbiodinium communities in waters, sediments, and corals of thermally distinct reef pools in American Samoa. PLoS One 10:e145099.

DOI 10.1371/journal.pone.0145099

De Barba M, Miquel C, Boyer F, Mercier C, Rioux D, Coissac E, and Taberlet P. 2014. DNA metabarcoding multiplexing and validation of data accuracy for diet assessment: application to omnivorous diet. Molecular Ecology Resources 14:306-323. DOI 10.1111/1755-0998.12188

DeBoer TS, Baker AC, Erdmann MV, Ambariyanto, Jones PR, and Barber PH. 2012. Patterns of Symbiodinium distribution in three giant clam species across the biodiverse Bird's Head region of Indonesia. Marine Ecology Progress Series 444:117-132. DOI 10.3354/meps09413

Decelle J, Carradec Q, Pochon X, Henry N, Romac S, Mahe F, Dunthorn M, Kourlaiev A, Voolstra CR, Wincker P, and de Vargas C. 2018. Worldwide occurrence and activity of the reefbuilding coral symbiont Symbiodinium in the open ocean. Current Biology 28:3625-3633. DOI 10.1016/j.cub.2018.09.024

Dubousquet V, Berteaux-Lecellier V, Lopes V, Raharivelomanana P, and Lecellier G. 2018. Existence of two novel and non-sectorized clades of the giant clam Tridacna maxima in French Polynesia: implications for connectivity and origin. HAL Archives Ouvertes:hal01713884.

Edgar RC. 2010. Search and clustering orders of magnitude faster than BLAST. Bioinformatics 26:2460-2461. DOI 10.1093/bioinformatics/btq461

Edgar RC. 2013. UPARSE: highly accurate OTU sequences from microbial amplicon reads. Nature Methods 10:996-998. DOI 10.1038/nmeth.2604

Edmunds PJ, Pochon X, Levitan DR, Yost DM, Belcaid M, Putnam HM, and Gates RD. 2014. Long-term changes in Symbiodinium communities in Orbicella annularis in St. John, US Virgin Islands. Marine Ecology Progress Series 506:129-144. 10.3354/meps 10808

Elbrecht V, and Steinke D. 2018. Scaling up DNA metabarcoding for freshwater macrozoobenthos monitoring. Freshwater Biology 64:380-387.

Peer) reviewing PDF | (2019:01:34199:1:2:NEW 28 Mar 2019) 
440

441

442

443

444

445

446

447

448

449

450

451

452

453

454

455

456

457

458

459

460

461

462

463

464

465

466

467

468

469

470

471

472

473

474

475

476

477

478

479

480

481

482

483

484

485

DOI 10.1111/fwb.13220

Engelbrektson A, Kunin V, Wrighton KC, Zvenigorodsky N, Chen F, Ochman H, and Hugenholtz P. 2010. Experimental factors affecting PCR-based estimates of microbial species richness and evenness. The International Society for Microbial Ecology Journal 4:642-647. DOI 10.1038/ismej.2009.153

Felsenstein J. 1985. Phylogenies and the Comparative Method. American Naturalist 125:1-15.

Fiore-Donno AM, Rixen C, Rippin M, Glaser K, Samolov E, Karsten U, Becker B, and Bonkowski M. 2018. New barcoded primers for efficient retrieval of cercozoan sequences in highthroughput environmental diversity surveys, with emphasis on worldwide biological soil crusts. Molecular Ecology Resources 18:229-239.

DOI 10.1111/1755-0998.12729

Fitt WK, and Trench RK. 1981. Spawning, development, and acquisition of zooxanthellae by Tridacna squamosa (mollusca, bivalvia). Biological Bulletin 161:213-235.

Franklin EC, Stat M, Pochon X, Putnam HM, and Gates RD. 2012. GeoSymbio: a hybrid, cloudbased web application of global geospatial bioinformatics and ecoinformatics for Symbiodinium-host symbioses. Molecular Ecology Resources 12:369-373. DOI 10.1111/j.1755-0998.2011.03081.x

Hall TA. 1999. BioEdit: a user-friendly biological sequence alignment editor and analysis program for Windows 95/98/NT. Nucleic Acids Research 41:95-98.

Hume BCC, Ziegler M, Poulain J, Pochon X, Romac S, Boissin E, de Vargas C, Planes S, Wincker P, and Voolstra CR. 2018. An improved primer set and amplification protocol with increased specificity and sensitivity targeting the Symbiodinium ITS2 region. PeerJ 6:e4816. DOI 10.7717/peerj.4816

Ikeda S, Yamashita H, Kondo SN, Inoue K, Morishima SY, and Koike K. 2017. Zooxanthellal genetic varieties in giant clams are partially determined by species-intrinsic and growthrelated characteristics. PLoS One 12:e0172285.

DOI 10.1371/journal.pone.0172285

Ikeda S, Yamashita H, Liao LM, and Koike K. 2016. A simple and rapid determination method for zooxanthellal genetic diversity in giant clams using multiplex PCR. Fisheries Science 82:747-753. DOI 10.1007/s12562-016-1004-X

Keeley N, Wood SA, and Pochon X. 2018. Development and preliminary validation of a multitrophic metabarcoding biotic index for monitoring benthic organic enrichment. Ecological Indicators 85:1044-1057.

DOI 10.1016/j.ecolind.2017.11.014

Klumpp D, and Griffiths C. 1994. Contributions of phototrophic and heterotrophic nutrition to the metabolic and growth requirements of four species of giant clam (Tridacnidae). Marine Ecology Progress Series 115:103-115.

Knop D. 1996. Giant clams - a comprehensive guide to the identification and care of tridacnid clams. Dähne Verlag, Ettlingen:p. 255.

Kozich JJ, Westcott SL, Baxter NT, Highlander SK, and Schloss PD. 2013. Development of a dual-index sequencing strategy and curation pipeline for analyzing amplicon sequence data on the MiSeq Illumina sequencing platform. Applied Environmental Microbiology 79:5112-5120. DOI 10.1128/AEM.01043-13

Kumar S, Stecher G, and Tamura K. 2016. MEGA7: Molecular Evolutionary Genetics Analysis version 7.0 for bigger datasets. Molecular Biology and Evolution 33:1870-1874. DOI 10.1093/molbev/msw054

PeerJ reviewing PDF | (2019:01:34199:1:2:NEW 28 Mar 2019) 
486

487

488

489

490

491

492

493

494

495

496

497

498

499

500

501

502

503

504

505

506

507

508

509

510

511

512

513

514

515

516

517

518

519

520

521

522

523

524

525

526

527

528

529

530

LaJeunesse T, Thornhill D, Cox E, Stanton F, Fitt W, and Schmidt G. 2004. High diversity and host specificity observed among symbiotic dinoflagellates in reef coral communities from Hawaii. Coral Reefs 23:596-603. DOI 10.1007/s00338-004-0428-4

LaJeunesse TC. 2001. Investigating the biodiversity, ecology, and phylogeny of endosymbiotic dinoflagellates in the genus symbiodinium using the its region: in search of a "species" level marker. Journal of Phycology 37:866-880.

LaJeunesse TC, Loh WKW, van Woesik R, Hoegh-Guldberg O, Schmidt GW, and Fitt WK. 2003. Low symbiont diversity in southern Great Barrier Reef corals, relative to those of the Caribbean. Limnology and Oceanography 48:2046-2054.

LaJeunesse TC, Parkinson JE, Gabrielson PW, Jeong HJ, Reimer JD, Voolstra CR, and Santos SR. 2018. Systematic revision of Symbiodiniaceae highlights the antiquity and diversity of coral endosymbionts. Current Biology 28:2570-2580 e2576.

DOI 10.1016/j.cub.2018.07.008

LaJeunesse TC, Smith R, Walther M, Pinzon J, Pettay DT, McGinley M, Aschaffenburg M, Medina-Rosas P, Cupul-Magana AL, Perez AL, Reyes-Bonilla H, and Warner ME. 2010. Host-symbiont recombination versus natural selection in the response of coraldinoflagellate symbioses to environmental disturbance. Proceedings of the Royal Society B 277:2925-2934. DOI 10.1098/rspb.2010.0385

Lee SY, Jeong HJ, Kang NS, Jang TY, Jang SH, and Lajeunesse TC. 2015. Symbiodinium tridacnidorum sp. nov., a dinoflagellate common to Indo-Pacific giant clams, and a revised morphological description of Symbiodinium microadriaticum Freudenthal, emended Trench \& Blank. European Journal of Phycology 50:155-172. DOI 10.1080/09670262.2015.1018336

List IR. 2018. The IUCN Red List of Threatened Species [WWW Document].

Macdonald AHH, Sampayo EM, Ridgway T, and Schleyer MH. 2008. Latitudinal symbiont zonation in Stylophora pistillata from southeast Africa. Marine Biology 154:209-217. DOI 10.1007/s00227-008-0915-1

Manning MM, and Gates RD. 2008. Diversity in populations of free-living Symbiodinium from a Caribbean and Pacific reef. Limnology and Oceanography 53:1853-1861.

Marcelino VR, and Verbruggen H. 2016. Multi-marker metabarcoding of coral skeletons reveals a rich microbiome and diverse evolutionary origins of endolithic algae. Scientific Reports 6:31508. DOI 10.1038/srep31508

Maruyama T, and Heslinga GA. 1997. Fecal discharge of zooxanthellae in the giant clam Tridacna derasa, with reference to their in situ growth rate. Marine Biology 127:473-477.

Mercier A, and Hamel JF. 1996. The secret of the giant clam. Freshwater and Marine Aquarium 19:112-113.

Neo ML, Eckman W, Vicentuan K, Teo SLM, and Todd PA. 2015. The ecological significance of giant clams in coral reef ecosystems. Biological Conservation 181:111-123. DOI 10.1016/j.biocon.2014.11.004

Pappas MK, He S, Hardenstine RS, Kanee H, and Berumen ML. 2017. Genetic diversity of giant clams (Tridacna spp.) and their associated Symbiodinium in the central Red Sea. Marine Biodiversity 47:1209-1222. DOI 10.1007/s12526-017-0715-2

Pochon X, Putnam HM, Burki F, and Gates RD. 2012. Identifying and characterizing alternative molecular markers for the symbiotic and free-living dinoflagellate genus Symbiodinium. PLoS One 7:e29816. DOI 10.1371/journal.pone.0029816 
531 Pochon X, Putnam HM, and Gates RD. 2014. Multi-gene analysis of Symbiodinium

532

533

534

535

536

537

538

539

540

541

542

543

544

545

546

547

548

549

550

551

552

553

554

555

556

557

558

559

560

561

562

563

564

565

566

567

568

569

570

571

572

573

574

575

576

dinoflagellates: a perspective on rarity, symbiosis, and evolution. PeerJ 2:e394. DOI 10.7717 peerj.394

Pochon X, Stat M, Takabayashi M, Chasqui L, Chauka LJ, Logan DDK, and Gates RD. 2010. Comparison of endosymbiotic and free-living Symbiodinium (Dinophyceae) diversity in a Hawaiian reef environment1. Journal of Phycology 46:53-65. DOI 10.1111/j.1529-8817.2009.00797.x

Rhodes J, Beale MA, and Fisher MC. 2014. Illuminating choices for library prep: a comparison of library preparation methods for whole genome sequencing of Cryptococcus neoformans using Illumina HiSeq. PLoS One 9:e113501.

DOI 10.1371/journal.pone.0113501

Romanek C, and Grossman EL. 1989. Stable isotope profiles of Tridacna maxima as environmental indicators. Palaios 4:402-413.

Shinzato C, Zayasu Y, Kanda M, Kawamitsu M, Satoh N, Yamashita H, and Suzuki G. 2018. Using seawater to document coral-zoothanthella diversity: a new approach to coral reef monitoring using environmental DNA. Frontiers in Marine Science 5:28. DOI 10.3389/fmars.2018.00028

Smith EG, Ketchum RN, and Burt JA. 2017. Host specificity of Symbiodinium variants revealed by an ITS2 metahaplotype approach. ISME J 11:1500-1503.

DOI 10.1038/ismej.2016.206

Stat M, Carter D, and Hoegh-Guldberg O. 2006. The evolutionary history of Symbiodinium and scleractinian hosts-Symbiosis, diversity, and the effect of climate change. Perspectives in Plant Ecology, Evolution and Systematics 8:23-43.

DOI 10.1016/j.ppees.2006.04.001

Stat M, Huggett MJ, Bernasconi R, DiBattista JD, Berry TE, Newman SJ, Harvey ES, and Bunce M. 2017. Ecosystem biomonitoring with eDNA: metabarcoding across the tree of life in a tropical marine environment. Scientific Reports 7:12240.

DOI 10.1038/s41598-017-12501-5

Takabayashi M, Adams LM, Pochon X, and Gates RD. 2011. Genetic diversity of free-living Symbiodinium in surface water and sediment of Hawai' $i$ and Florida. Coral Reefs 31:157167. DOI 10.1007/s00338-011-0832-5

Thomas L, Kendrick GA, Kennington WJ, Richards ZT, and Stat M. 2014. Exploring Symbiodinium diversity and host specificity in Acropora corals from geographical extremes of Western Australia with 454 amplicon pyrosequencing. Molecular Ecology 23:3113-3126. DOI 10.1111/mec.12801

Trench R, Wethey D, and Porter J. 1981. Observations on the symbiosis with zooxanthellae among the tridacnidae (Mollusca, Bivalvia). Biological Bulletin 161:180-198.

von Ammon U, Wood SA, Laroche O, Zaiko A, Tait L, Lavery S, Inglis G, and Pochon X. 2018. The impact of artificial surfaces on marine bacterial and eukaryotic biofouling assemblages: A high-throughput sequencing analysis. Marine Environmental Research 133:57-66. DOI 10.1016/j.marenvres.2017.12.003

Watanabe T, Suzuki A, Kawahata H, Kan H, and Ogawa S. 2004. A 60-year isotopic record from a mid-Holocene fossil giant clam (Tridacna gigas) in the Ryukyu Islands: physiological and paleoclimatic implications. Palaeogeography, Palaeoclimatology, Palaeoecology 212:343-354.

DOI 10.1016/s0031-0182(04)00358-x

Peer) reviewing PDF | (2019:01:34199:1:2:NEW 28 Mar 2019) 
577 Weber M. 2009. The Biogeography and Evolution of Symbiodinium in Giant Clams (Tridacnidae). $578 \quad$ PhD Dissertation, UC Berkeley:103 pages + appendices.

579 Wecker P, Fournier A, Bosserelle P, Debitus C, Lecellier G, and Berteaux-Lecellier V. 2015. 580 Dinoflagellate diversity among nudibranchs and sponges from French Polynesia: insights into associations and transfer. Comptes Rendus Biologies 338:278-283. DOI 10.1016/j.crvi.2015.01.005

Zaiko A, Schimanski K, Pochon X, Hopkins GA, Goldstien S, Floerl O, and Wood SA. 2016. Metabarcoding improves detection of eukaryotes from early biofouling communities: implications for pest monitoring and pathway management. Biofouling 32:671-684. DOI 10.1080/08927014.2016.1186165

Zhang GK, Chain FJJ, Abbott CL, and Cristescu ME. 2018. Metabarcoding using multiplexed markers increases species detection in complex zooplankton communities. Evolutionary Applications 11:1901-1914. DOI 10.1111/eva.12694

590 


\section{Table 1 (on next page)}

DNA sequence counts following demultiplexing of 'Pooled' and 'Controls' samples.

Number of DNA sequences recovered from each sample (S141-S152), before and after quality filtration, and after demultiplexing into each gene. Samples S141 to S143 were used as control samples, each targeting only one of three PCR amplicons. Columns highlighted in grey show a low background contamination. 
1 Table 1 Number of DNA sequences recovered from each sample (S141-S152), before and after quality 2 filtration, and after demultiplexing into each gene. Samples S141 to S143 were used as control samples, 3 each targeting only one of three PCR amplicons. Columns highlighted in grey show a low background 4 contamination.

\begin{tabular}{llllll}
\hline $\begin{array}{l}\text { Sample } \\
\text { ID }\end{array}$ & $\begin{array}{l}\text { Source } \\
\text { reads }\end{array}$ & $\begin{array}{l}\text { Filtered } \\
\text { reads }\end{array}$ & reads & $\begin{array}{l}\text { ITS2 } \\
\text { reads }\end{array}$ & LSU \\
reads
\end{tabular}

\section{Controls}

ITS2 only

$\begin{array}{llllll}\text { S141 } & 85824 & 52588 & 8 & 52335 & 1 \\ \text { S142 } & 81924 & 52270 & 10 & 51988 & 6 \\ \text { S143* } & 130 & 13 & 5 & 6 & 2\end{array}$

LSU only

$\begin{array}{lllllll}\text { S141 } & 56565 & 31134 & 8 & 7 & 30758 \\ \text { S142 } & 92110 & 62629 & 23 & 0 & 62129 \\ \text { S143 } & 114431 & 69823 & 9 & 0 & 69318\end{array}$

23S only

\begin{tabular}{llllll} 
S141 & 77522 & 66763 & 66399 & 3 & 3 \\
S142 & 42004 & 36422 & 36263 & 3 & 9 \\
S143 & 27894 & 24239 & 24149 & 1 & 3 \\
\hline
\end{tabular}

Total reads 15900471104687398442339780359768

5 *One control sample (S143 ITS2) failed at sequencing, resulting in only 130 raw reads. 6 


\section{Table 2 (on next page)}

Percentage comparison of each Symbiodiniaceae sub-generic genotype per gene per treatment.

Percentage comparison of each Symbiodiniaceae sub-generic genotype recovered using the three amplicon markers in 'Pooled' versus single 'Control' markers (see Table 1). The proportion of each sub-generic type between 'Pooled' and 'Control' is almost identical for the $23 S$ marker, but shows some minor differences for the ITS2 and LSU markers (grey shades). 
Table 2 Percentage comparison of each Symbiodiniaceae sub-generic genotype recovered using the three amplicon markers in 'Pooled' versus single 'Control' markers (see Table 1). The proportion of each sub-generic type between 'Pooled' and 'Control' is almost identical for the $23 S$ marker, but shows some minor differences for the ITS2 and LSU markers (grey shades).

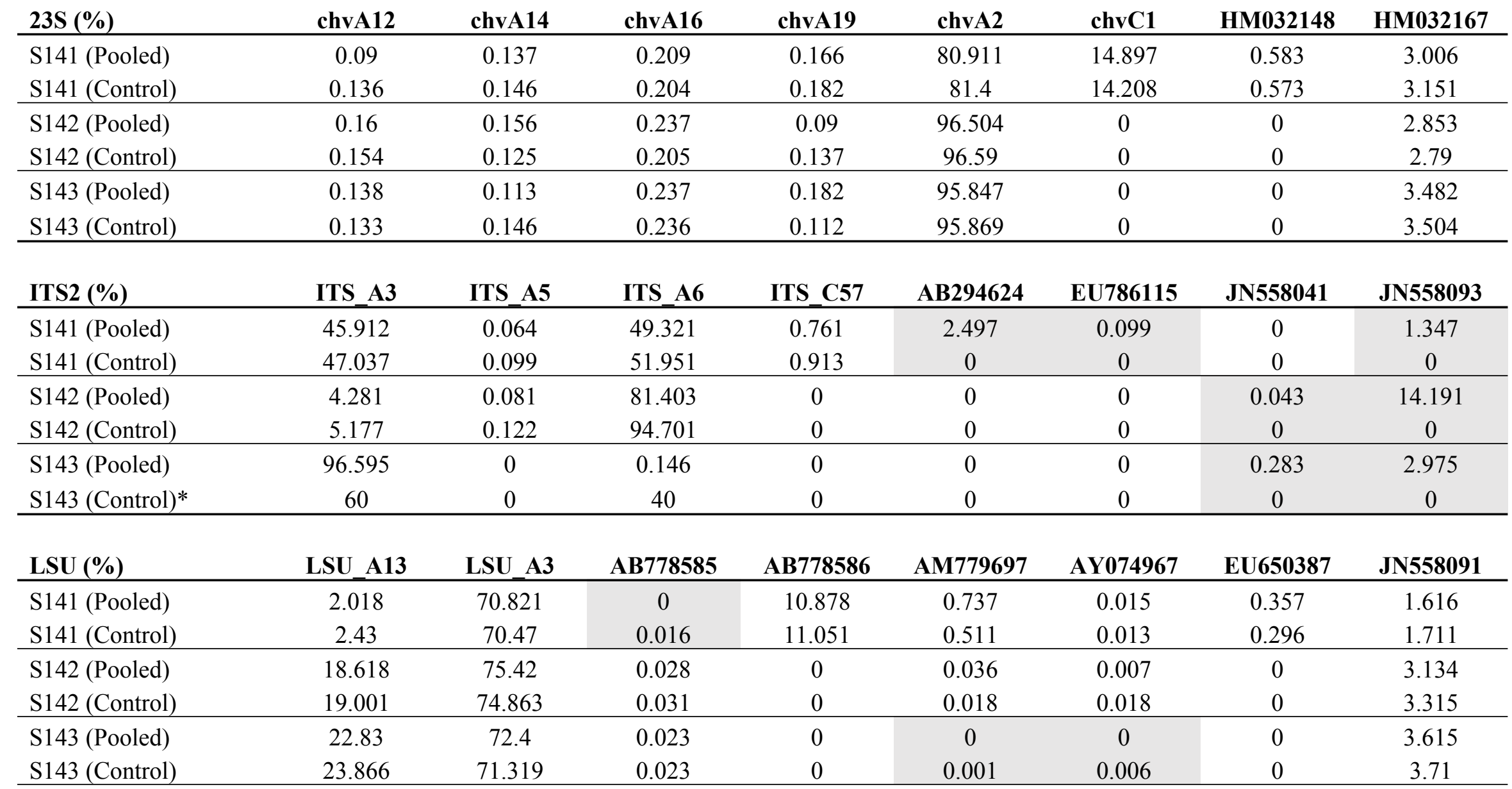




\begin{tabular}{lccccccc} 
LSU (\%) - continued & JN558092 & JN558093 & JN558096 & JN665089 & KC510080 & KT179776 & LK934674 \\
\hline S141 (Pooled) & 0 & 0.447 & 0.007 & 0.06 & 9.657 & 2.174 & 1.214 \\
S141 (Control) & 0.01 & 0.485 & 0.023 & 0.039 & 9.539 & 2.202 & 1.204 \\
\hline S142 (Pooled) & 0.014 & 0.264 & 0 & 0 & 0 & 0 & 2.479 \\
S142 (Control) & 0.019 & 0.261 & 0.002 & 0 & 0 & 0 & 2.474 \\
\hline S143 (Pooled) & 0.006 & 0.173 & 0.006 & 0 & 0 & 0 & 0.947 \\
S143 (Control) & 0.009 & 0.234 & 0.001 & 0 & 0 & 0 & 0.831 \\
\hline
\end{tabular}

*One control sample (S143 ITS2 only) failed the sequencing resulting in only 130 raw reads (see Table 1). 
Figure 1

Sampling sites.

Location and sample identification for the twelve Tridacna maxima samples investigated in this study (credit to R. Canavesio). 


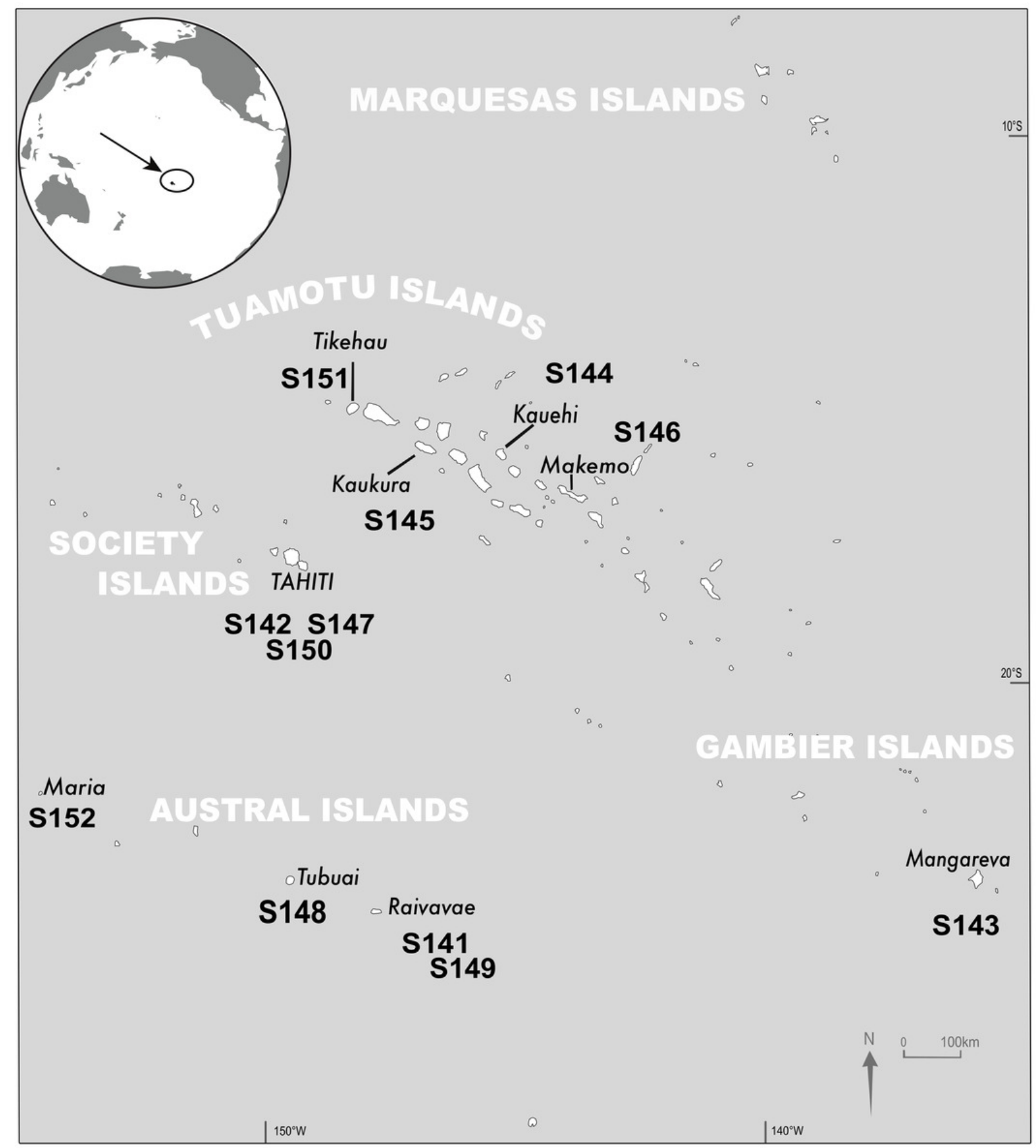


Figure 2

Proportion and diversity of Symbiodiniaceae genotypes across markers.

Global Symbiodiniaceae diversity charts obtained from each of the three datasets: A) 23S, B) ITS2, and C) LSU). The proportion of sequences matching one of the three in-house reference databases or NCBI (inner circles) and their corresponding phylogenetic affiliation at genus (i.e clade; middle circles) and sub-generic (i.e. subclade; outer circles) levels. Sequence reads representing $<0.1 \%$ of total read abundance are not included. 


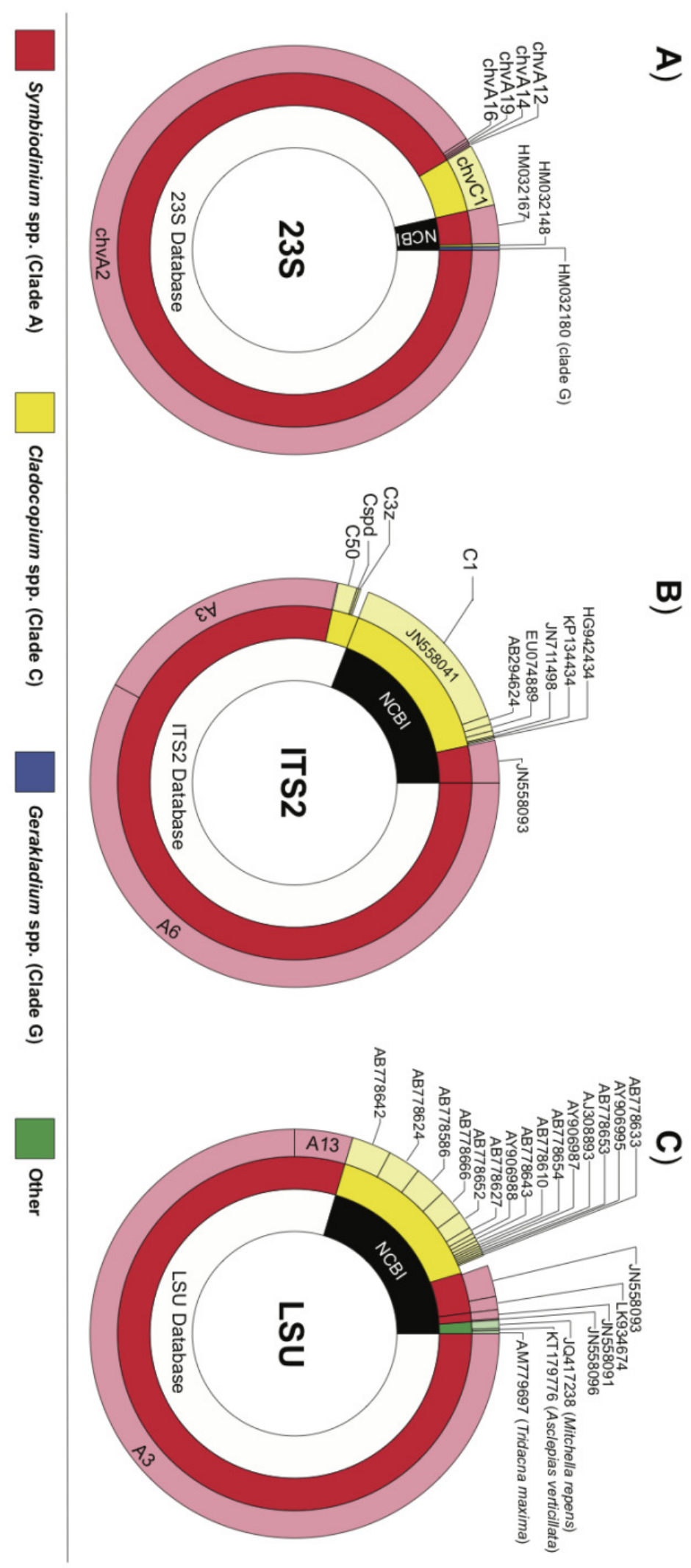

